\title{
Ultrasonographic evaluation of submucosal thickness in oral submucous fibrosis patients: a cross-sectional study
}

\author{
Aditya Dupare ${ }^{A, B, C, D, E, F}$, Apeksha Dhole ${ }^{E}$ \\ V.S.P.M. Dental College and Research Center, Nagpur, India
}

\section{Abstract}

Purpose: To evaluate the role of ultrasonography in oral submucous fibrosis (OSMF) patients.

Material and methods: A total of 150 subjects were divided equally into six groups (Group I: 25 healthy subjects; Group II: 25 healthy subjects with habit; Group III: 25 OSMF stage I; Group IV: 25 OSMF stage II; Group V: 25 OSMF stage III; and Group VI: 25 stage OSMF IVA). The grading of OSMF were done according the clinical classification given by Khanna and Andrade (2005). After fulfilling inclusion and exclusion criteria each subject underwent extraoral ultrasonographic evaluation of submucosal thickness and vascularity in terms of peak systolic velocity (PSV), bilaterally on buccal and labial mucosa. Furthermore, statistical comparison of each group was done, and sensitivity and specificity of USG measurements was obtained in comparison with clinical diagnosis. The statistical analysis was performed using SPSS ver. 20.0.

Results: A statistically significant increase in mean submucosal thickness was shown, and a decrease in PSV with the advancement of severity of the OSMF. In ultrasonographic diagnosis of OSMF, the reported submucosal thickness had a sensitivity, specificity, PPV, NPV, and accuracy was $80 \%, 100 \%, 100 \%, 71.4 \%$, and $87 \%$, respectively, but PSV failed to classify the lesion.

Conclusions: Because the severity of the disease showed a direct relationship with submucosal thickness and an inverse relationship with PSV, habit-induced mucosal alteration in submucosal thickness can be seen on USG, which cannot be appreciated on clinical examination. Hence, USG can be a promising tool for early diagnosis, assessment of the severity, and evaluation of prognosis of OSMF.

Key words: OSMF, ultrasonography (USG), submucosal thickness, peak systolic velocity (PSV).

\section{Introduction}

Oral submucous fibrosis (OSMF) is one of the most common premalignant conditions seen in the Indian population. It affects all parts of the oral cavity including buccal mucosa, labial mucosa, tongue, and soft and hard palate. The aetiology of OSMF is multifactorial, but areca nut and its products are considered to be the main causative agents. The condition is characterised by a burning sensation, blanching, and stiffness of the oral mucosa causing reduction in mouth opening [1]. Clinical examination alone may be subjective and is probably insufficient to characterise the disease severity adequately. Biopsy, being a minor surgical procedure, has its limitations, e.g. more time consuming, causes discomfort to patients, and tissue obtained from any single site may not be representative of the true extent of the disease [2].

USG imaging is a safe, readily available, non-invasive, non-ionising, and cost-effective real-time modality that can be used to image superficial tissues. Because of its non-invasive nature and safety, it has better patient acceptance. In addition, because of the wider area that can be imaged, USG may a valuable tool to determine the extent and severity of the disease as well as to monitor the

Correspondence address:

Dr. Aditya Dupare, V.S.P.M. Dental College and Research Center, Digdoh Hill, Hingna Road, 440019 Nagpur, India, phone: +91 9665559075,

e-mail: adupare@ymail.com

Authors' contribution:

A Study design · B Data collection · C Statistical analysis · D Data interpretation · E Manuscript preparation · F Literature search · G Funds collection 
response to the treatment, thus supplementing clinical and histological details [3-5]. The use of USG in the evaluation of OSMF draws inspiration from its application in scleroderma, where USG has been used for diagnosis as well as follow-up, based on the alteration in the thickness and the echogenicity of the dermis. OSMF is similar in many aspects to scleroderma, where continuous and uncontrolled fibrosis accompanies a chronic inflammatory reaction. Manjunath et al. reported that USG can delineate feeble fibrotic bands in clinically normal appearing buccal mucosa in OSMF patients. They also show decreased vascularity (PSV) in the affected area. Thus, patients with poor vascularity in the affected area responded poorly to treatment, suggesting the need to alter the treatment schedule $[4,6,7]$.

The diagnostic accuracy of USG is found to be useful in the diagnosis of various malignant tumours, periapical pathologies, and TMJ disorders. Very few studies have reported in literature with the use of USG in OSMF, and to the best of our knowledge, not a single study has been performed to show its diagnostic potential in OSMF [8].

Considering the need for early detection of OSMF, we aimed to conduct a study to evaluate the role of USG in OSMF using USG parameters, i.e. submucosal thickness and PSV bilaterally on buccal and labial mucosa, and compared it to healthy individuals with or without habits.

\section{Material and methods}

This is a hospital-based, cross-sectional, observational study that was initiated after approval from the Institutional Ethics Committee, and it was carried out in the Department of Oral Medicine and Radiology and Department of Radiodiagnosis. In the study group, OSMF patients and patients with a habit were included while patients with previous history with the management of OSMF and coexisting lesion with OSMF were excluded from the study. A total of 150 subjects were selected randomly from the departmental OPD and categorised in six groups of 25 subjects: Group I - healthy patients, Group II - healthy patients with habit, Group III - OSMF stage I, Group IV - OSMF stage II, Group V - OSMF stage III and Group VI - OSMF stage IVA.

After obtaining informed consent from all the subjects, detailed clinical history was recorded and clinical examination was carried out in a dental chair under proper illumination. Patients with OSMF were graded clinically according to the classification system given by Khanna and Andrade in 1995 [9]. All the subjects were divided into six groups as per the criteria. All the subjects then underwent USG examination. The submucosal thickness was measured in B-Mode while peripheral arterial flow was examined, and peak systolic velocity (PSV) was measured in colour Doppler (D-Mode) for both buccal and labial mucosa. The patient was made to lie supine on the examination table with the shoulders supported by a pillow and

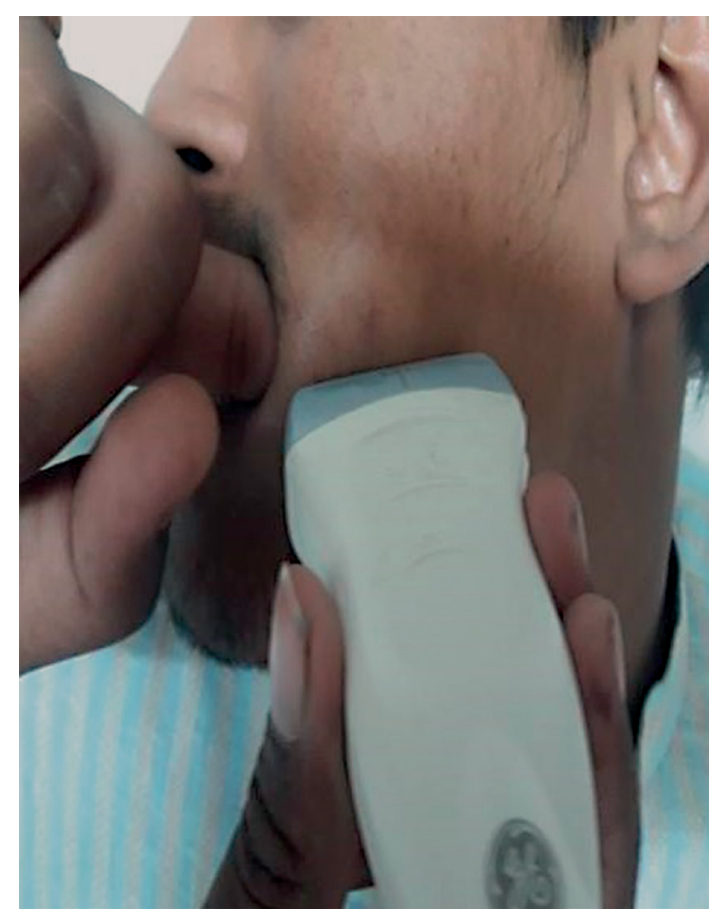

Figure 1. Position of transducer for the buccal mucosa

the operator seated on the right side. The coupling gel was applied on the buccal and labial surfaces extra orally. Subjects were instructed to place their index finger intraorally against the lining mucosa to delineate the empty spaces of the oral cavity during the scan. Transcutaneous imaging of the right and left buccal mucosa (RBM and LBM) and upper and lower labial mucosa (ULM and LLM) was performed by a single trained general radiologist. For buccal mucosa, the transducer probe was placed at the level of the occlusal line (Figure 1). For upper and lower labial mucosa the transducer probe was placed in the region of the philtrum and mentalis, respectively (Figures 2A and $2 \mathrm{~B}$ ).

Submucosal thickness measurements in millimetres $(\mathrm{mm})$ were taken at three points, i.e. the anterior (D1), middle (D2), and posterior (D3) for buccal mucosa. Similarly, for labial mucosa the measurements were taken at right (D1), middle (D2), and left (D3) points. The mean submucosal thickness of three points (D1, D2, and D3) was calculated for buccal and labial mucosa. Figure 3 shows USG images of submucosal thickness in centimetres of all six groups. The USG settings were shifted to D-mode to calculate peripheral arterial PSV of buccal and labial mucosa. It was calculated along the hypoechoic band of submucosa and was recorded at the highest point of the waveform, which is represented as the volume (V1) in centimetres/second ( $\mathrm{cm} / \mathrm{s})$. Figure 4 shows USG images of PSV in $\mathrm{cm} / \mathrm{s}$ of all six groups. The data were summarised in terms of statistical parameters including mean, standard deviation, and range. The buccal and labial sub-mucosal thickness on either side was obtained and summarised in terms of mean and standard deviation according to groups. One-way analysis of variance 


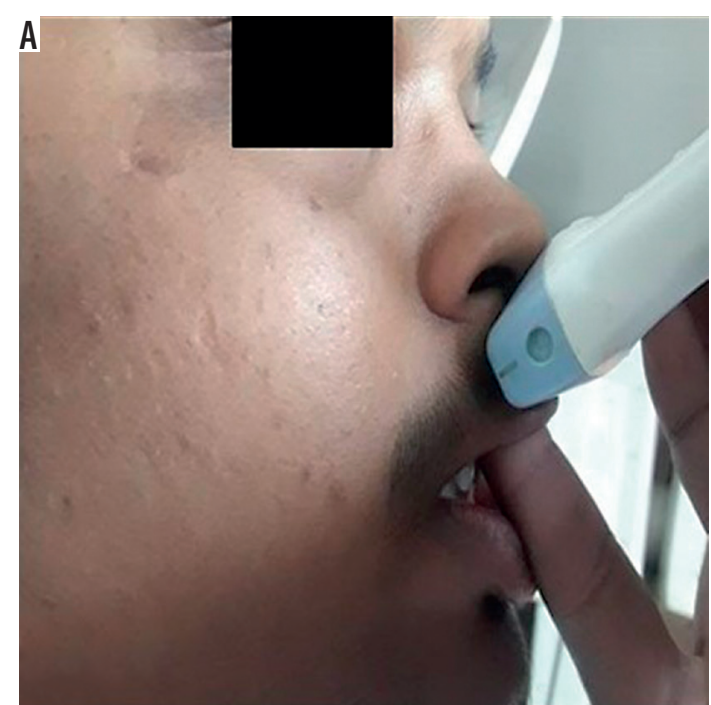

Figure 2. Position of transducer for upper (A) and lower (B) labial mucosa

(ANOVA) was performed to determine the significance of difference of means across groups. Pairwise analysis of means was performed using Tukey's post-hoc test. Similar analyses were performed for the parameter PSV. The sensitivity and specificity of USG measurements was obtained in comparison with clinical diagnosis. The analyses were performed using SPSS ver. 20.0.

\section{Results}

This hospital-based study included a total of 150 subjects divided into six equal groups. The condition of the the subjects was diagnosed clinically and ultrasonographically. On statistical analysis the age range for controls with and without habit was 19-48 years, and amongst 100 cases of OSMF there was a male predominance and the age range was 20-44 years (mean age 32 years).

The descriptive statistics for average sub mucosa thickness across six different stages of OSMF for RBM, LBM, ULM, and LLM. The comparison of mean thickness across groups for both buccal and labial mucosa was performed using one-way ANOVA. For RBM, the mean thickness across groups differed highly significantly across groups as indicated by $p$-value $<0.0001$. Further on the left side also, the average thickness was significantly different across groups with $p$-value $<0.0001$. As regards the thickness of ULM, the difference of average thickness was significant with $p$-value $<0.0001$, and on LLM the average thickness was significant with $p$-value $<0.0001$.

The pairwise comparison of average thickness of LBM between groups was performed using Tukey's post-hoc test. It is evident from the table that all the paired comparisons showed highly significant difference of means $(p<0.0001)$, except Group II and III $(p=0.4573)$. Pairwise comparison of average thickness of ULM between groups was performed using Tukey's post-hoc test. It is evident from the table that all the paired comparisons showed highly significant difference of means $(p<0.0001)$,

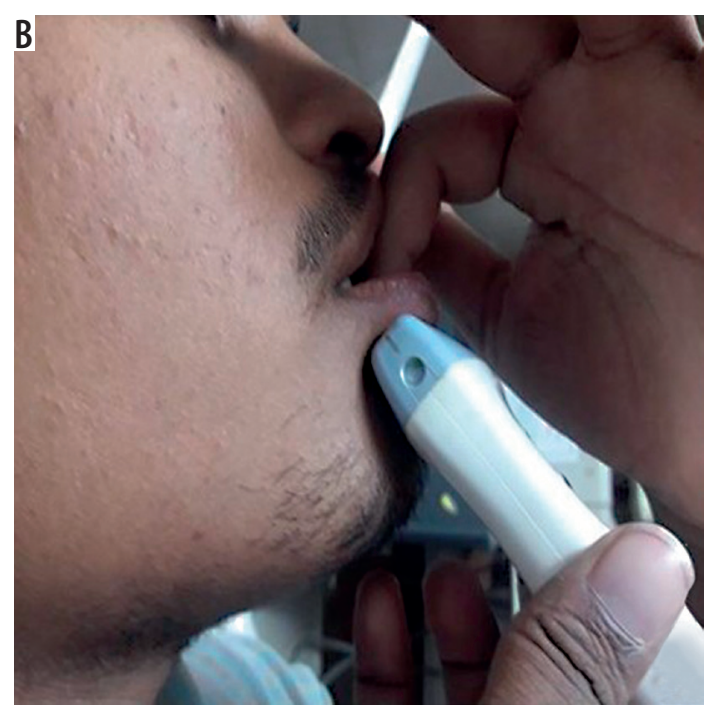

except Group II and III ( $p=0.9998)$. Pairwise comparison of average thickness of LLM was performed using Tukey's post-hoc test. It is evident from the table that all the paired comparisons showed highly significant difference of means $(p<0.0001)$, except Group II and III $(p=0.9665)$ (Table 1$)$.

The descriptive statistics of PSV for RBM and LBM as well as ULM and LLM across different groups of OSMF. The comparison of mean PSV across groups was performed using one-way ANOVA for both buccal and labial mucosa for each side. For RBM, the difference of mean PSV across groups was highly significant with $p$-value $<0.0001$. Further on LBM, the difference of mean PSV across stages was also significant $(p<0.0001)$. As regards ULM, the mean PSV across groups also showed statistical significance with $p$-value $<0.0001$, and a similar observation was made for LLM.

The pairwise comparison of mean PSV of RBM was performed using Tukey's test. The analysis revealed that the means differed significantly for all comparisons $(p<0.0001)$, except for the comparison Group V and Group VI with $p$-value of $0.9992(p>0.05)$. The pairwise comparison of mean PSV of buccal mucosa for left side was performed using Tukey's test. The analysis revealed that the means differed significantly for all comparisons $(p<0.0001)$, except for the comparison between Group V and Group VI with a $p$-value of $0.9999(p>0.05)$. The pairwise comparison of mean PSV of ULM using Tukey's test. The analysis revealed that the means differed significantly for all comparisons $(p<0.0001)$, except for the comparison Group I and Group II with $p$-value of 0.9999 $(p>0.05)$ and Group V and Group VI with $p$-value of $0.8599(p>0.05)$. The pairwise comparison of mean PSV of LLM using Tukey's test. The analysis revealed that the means differed significantly for all comparisons $(p<0.0001)$, except for the comparison Group I and Group II with $p$-value of $0.6026(p>0.05)$ and Group V and Group VI with $p$-value of $0.2759(p>0.05)$ (Table 2). 
Group I (healthy subjects)

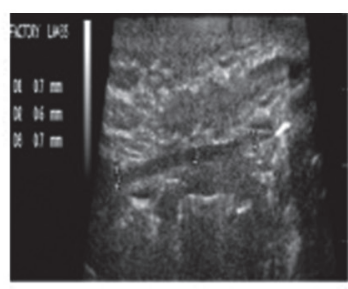

RBM

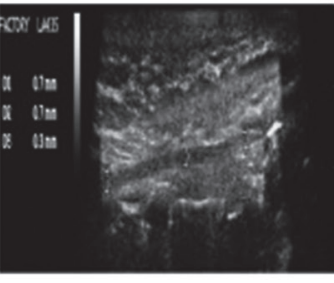

RBM

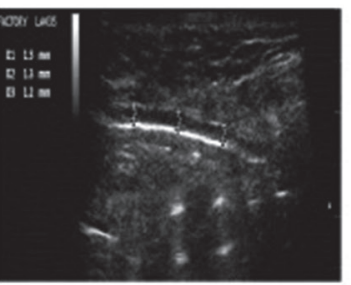

RBM

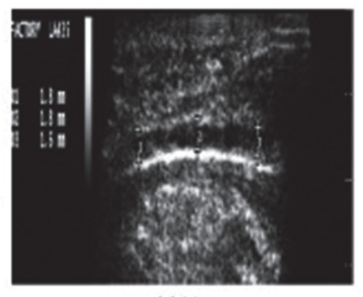

RBM

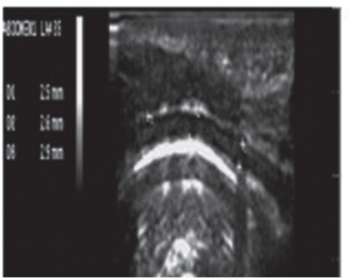

RBM

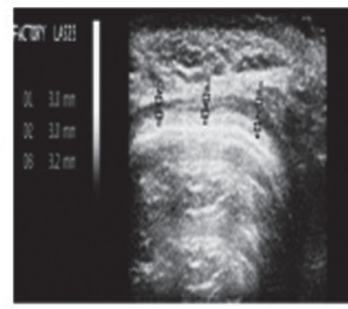

RBM

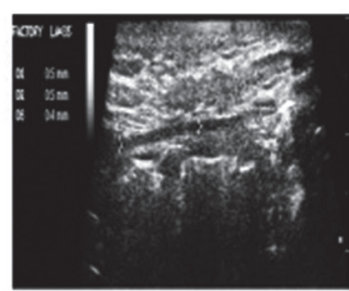

LBM

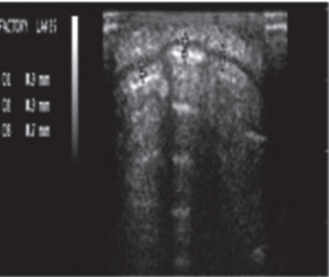

ULM

Group II (healthy subjects with habit)

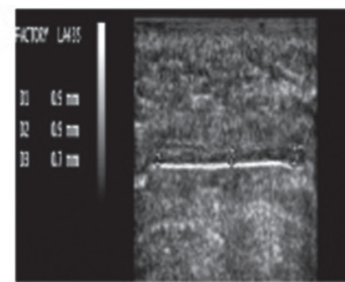

LBM

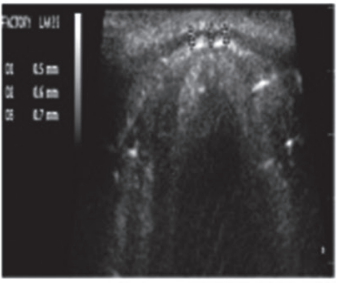

ULM

Group III (OSMF stage I)

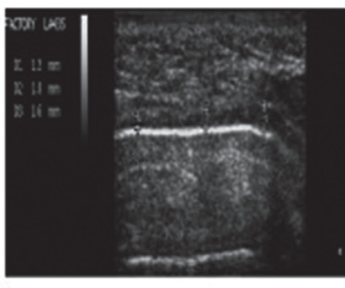

LBM

Group IV (OSMF stage II)

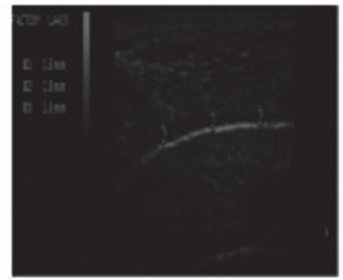

LBM

Group V (OSMF stage III)

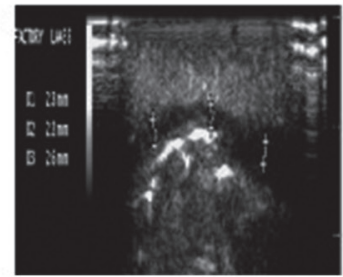

LBM

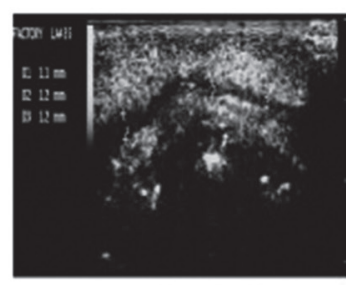

ULM

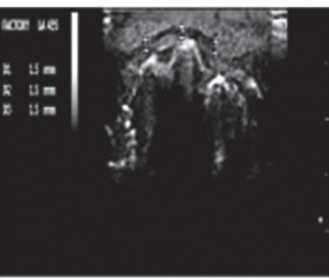

ULM

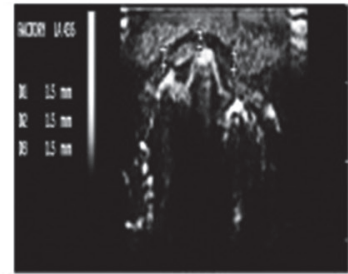

ULM

Group VI (OSMF stage IV)

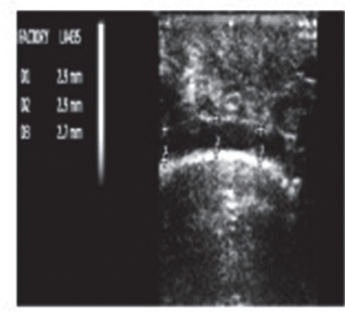

LBM

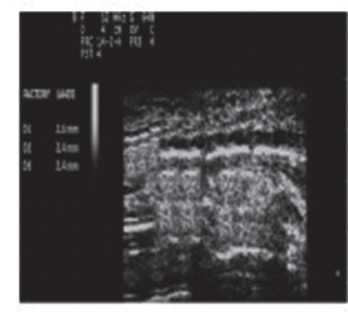

ULM

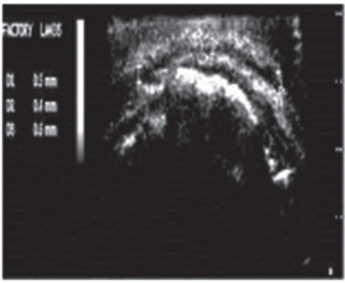

LLM

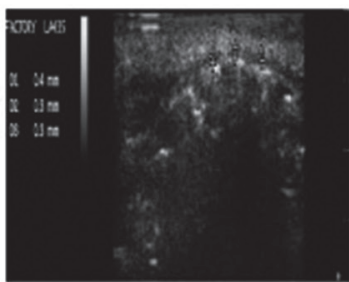

LLM

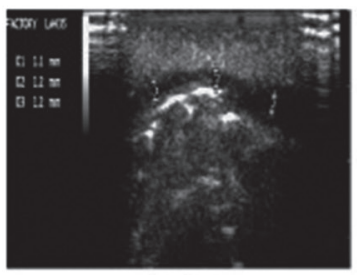

LLM

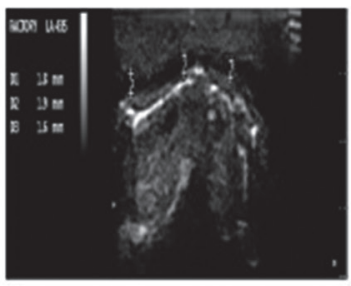

LLM

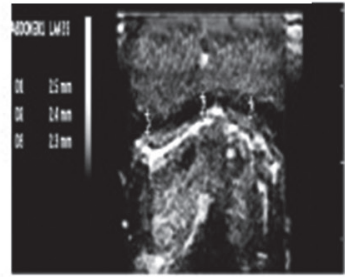

LLM

Figure 3. Ultrasonography images of submucosal thickness in cm of all six groups

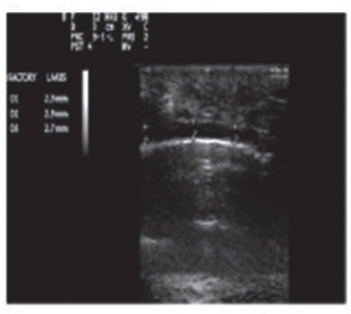

LLM 
Group I (healthy subjects)

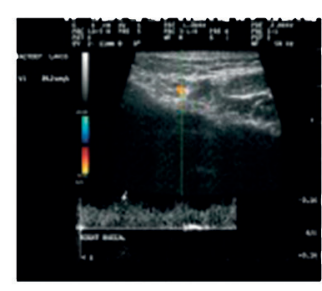

RBM

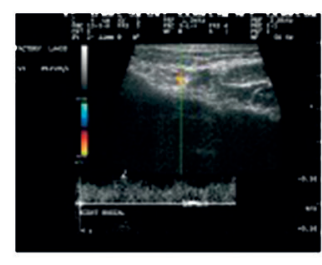

RBM

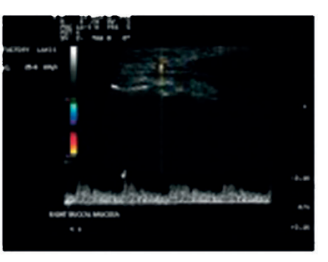

RBM

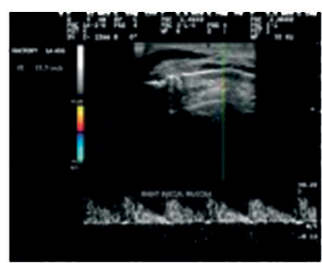

RBM

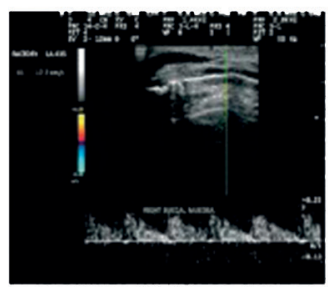

RBM

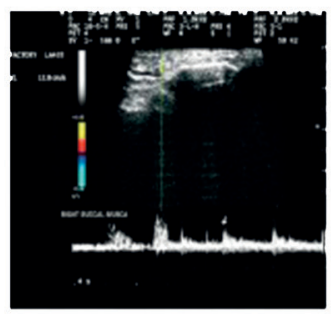

RBM

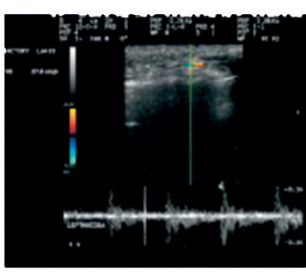

LBM

Group II (healthy subjects with habit)

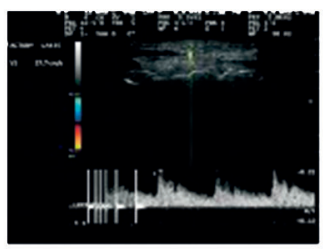

LBM

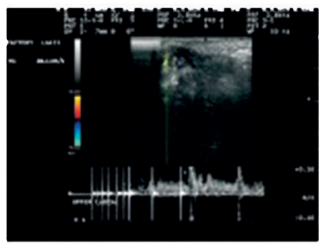

ULM

Group III (OSMF stage I)

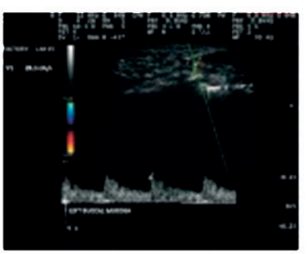

LBM

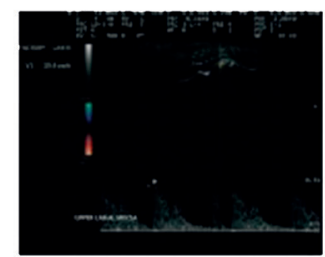

ULM

Group IV (OSMF stage II)

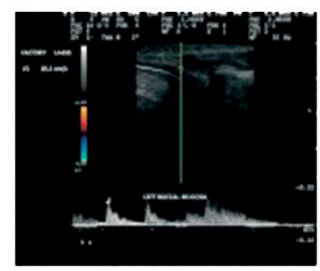

LBM

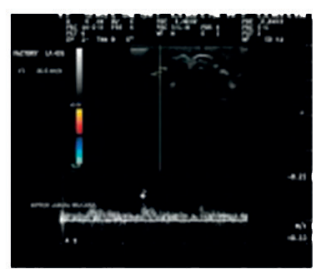

ULM

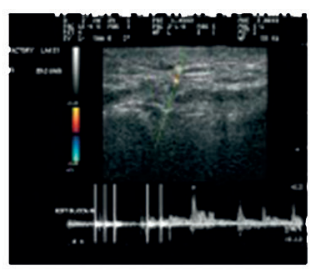

LLM

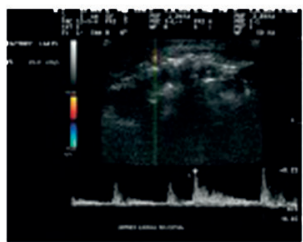

LLM

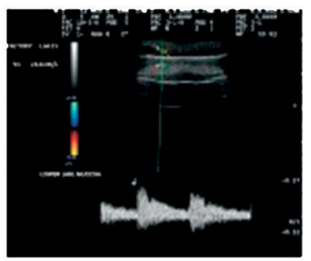

LLM

Group V (OSMF stage III)

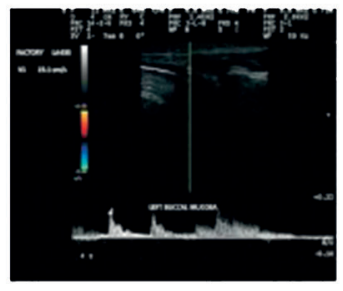

LBM

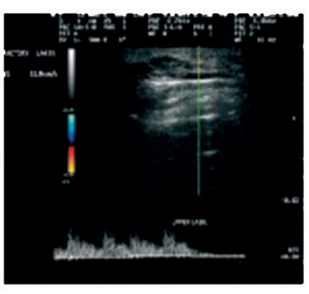

ULM

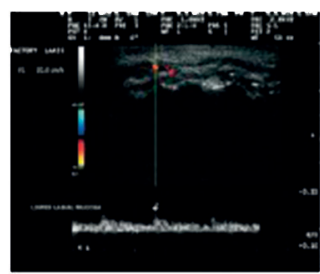

LLM

Group VI (OSMF stage IVa)

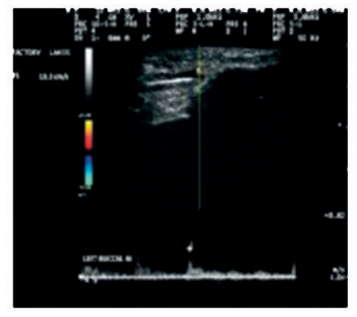

LBM

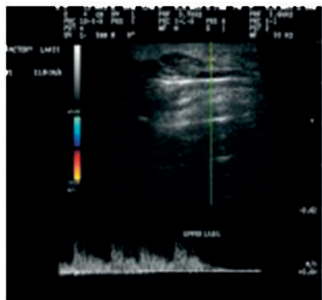

ULM

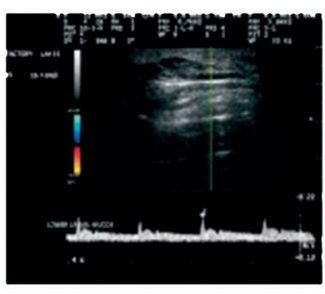

LLM

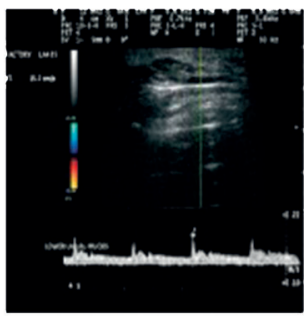

LLM

Figure 4. Ultrasonography images of peak systolic velocity (PSV) in cm/s of all six groups 
Table 1. Mean and SD of average sub mucosal thickness across six different groups for right and left buccal mucosa, and upper and lower labial mucosa

\begin{tabular}{|l|c|c|c|c|}
\multirow{2}{*}{ Group } & \multicolumn{2}{|c|}{ Thickness - buccal mucosa in mm (mean \pm SD) } & \multicolumn{2}{c|}{ Thickness - labial mucosa in mm (mean \pm SD) } \\
\cline { 2 - 5 } & Right & Left & Upper & Lower \\
\hline I & $0.50 \pm 0.115^{\mathrm{a}}$ & $0.62 \pm 0.173^{\mathrm{a}}$ & $0.58 \pm 0.144^{\mathrm{a}}$ & $0.52 \pm 0.138^{\mathrm{a}}$ \\
\hline II & $1.27 \pm 0.112^{\mathrm{b}}$ & $1.21 \pm 0.109^{\mathrm{b}}$ & $1.24 \pm 0.169^{\mathrm{b}}$ & $1.28 \pm 0.127^{\mathrm{b}}$ \\
\hline III & $1.28 \pm 0.135^{\mathrm{b}}$ & $1.31 \pm 0.105^{\mathrm{b}}$ & $1.26 \pm 0.122^{\mathrm{b}}$ & $1.33 \pm 0.147^{\mathrm{b}}$ \\
\hline V V & $1.65 \pm 0.107^{\mathrm{c}}$ & $1.66 \pm 0.118^{\mathrm{c}}$ & $1.61 \pm 0.109^{\mathrm{c}}$ & $1.63 \pm 0.095^{\mathrm{c}}$ \\
\hline VI & $2.35 \pm 0.129^{\mathrm{d}}$ & $2.33 \pm 0.129^{\mathrm{d}}$ & $2.30 \pm 0.191^{\mathrm{d}}$ & $2.27 \pm 0.154^{\mathrm{d}}$ \\
\hline$p$-value* & $3.12 \pm 0.439^{\mathrm{e}}$ & $3.01 \pm 0.337^{\mathrm{e}}$ & $2.93 \pm 0.324^{\mathrm{e}}$ & $2.85 \pm 0.386^{\mathrm{e}}$ \\
\hline
\end{tabular}

*0btained using one-way ANOVA. HS - highly significant. Similar superscripts in each vertical column indicate statistical insignificance using Tukey's post-hoc test.

Table 2. Mean and SD of PSV for buccal and labial mucosa according to groups of OSMF and sides

\begin{tabular}{|l|c|c|c|c|}
\hline \multirow{2}{*}{ Group } & \multicolumn{2}{|c|}{ Buccal mucosa (mean \pm SD) } & \multicolumn{2}{c|}{ Labial mucosa (mean \pm SD) } \\
\cline { 2 - 5 } & Right & Left & Upper & $26.28 \pm 1.749^{\mathrm{a}}$ \\
\hline I II & $29.10 \pm 0.849^{\mathrm{a}}$ & $29.38 \pm 1.648^{\mathrm{a}}$ & $26.98 \pm 1.3^{\mathrm{a}}$ & $26.99 \pm 0.853^{\mathrm{a}}$ \\
\hline III & $27.11 \pm 1.098^{\mathrm{b}}$ & $26.63 \pm 1.365^{\mathrm{b}}$ & $26.93 \pm 1.090^{\mathrm{a}}$ & $22.60 \pm 1.303^{\mathrm{b}}$ \\
\hline IV & $23.00 \pm 1.147^{\mathrm{c}}$ & $23.26 \pm 1.233^{\mathrm{c}}$ & $22.92 \pm 1.286^{\mathrm{b}}$ & $17.15 \pm 1.459^{\mathrm{c}}$ \\
\hline V & $17.36 \pm 1.564^{\mathrm{d}}$ & $16.55 \pm 1.233^{\mathrm{d}}$ & $16.86 \pm 1.693^{\mathrm{c}}$ & $14.56 \pm 1.500^{\mathrm{d}}$ \\
\hline VI & $14.52 \pm 1.680^{\mathrm{e}}$ & $14.45 \pm 1.471^{\mathrm{e}}$ & $14.67 \pm 1.806^{\mathrm{d}}$ & $13.60 \pm 2.249^{\mathrm{d}}$ \\
\hline$p$-value* & $14.35 \pm 2.886^{\mathrm{e}}$ & $14.40 \pm 1.758^{\mathrm{e}}$ & $14.17 \pm 1.994^{\mathrm{d}}$ & $<0.0001$ (HS) \\
\hline
\end{tabular}

*0btained using one-way ANOVA. HS - highly significant. Similar superscripts in each vertical column indicate statistical insignificance using Tukey's post-hoc test.

Table 3. Distribution of patients in two groups according to optimal thickness cut-offs

\begin{tabular}{l|c|c|}
$\begin{array}{l}\text { Sub-mucosal thickness (average of left } \\
\text { and right) }\end{array}$ & OSMF & Control \\
\hline$\geq 1.37$ & 80 & 0 \\
\hline$<1.37$ & 20 & 50 \\
\hline $\begin{array}{l}\text { Sub-mucosal thickness (average of upper } \\
\text { and lower side) }\end{array}$ & OSMF & Control \\
\hline$\geq 1.47$ & 76 & 0 \\
\hline$<1.47$ & 24 & 50 \\
\hline
\end{tabular}

The sensitivity and specificity of USG parameters, i.e. sub-mucosal thickness, was determined referring to clinical diagnosis. ROC analysis was performed on both the parameters independently by classifying observations as per clinical diagnosis. As regards clinical diagnosis, patients from Group I and II were regarded as normal, while those from Groups III to VI were regarded as cases. The data on left and right side was averaged to generate a single array of thickness values. The array was split into two groups as per clinical diagnosis. ROC analysis resulted into an area under curve (AUC) of 0.957 indicating excellent accuracy of sub-mucosal thickness in classifying cases. The Youden index based on sensitivity and specificity at each value of parameter was obtained. The maximum index corresponded to a thickness value of 1.37 . This cut-off resulted in a sensitivity of $0.8(80 \%)$, and specificity of $1(100 \%)$. The positive predictive value (PPV) was 1 (100\%), while the negative predictive value (NPV) was 0.714 (71.4\%). The accuracy of the parameter in diagnosis was $87 \%$.

On similar lines, the upper and lower sub-mucosal thickness was averaged resulting in a single array of thickness values. The ROC analysis resulted in an AUC of 0.949 indicating excellent accuracy of sub-mucosal thickness in classifying cases. The Youden index resulted into a cutoff value thickness of 1.47 resulting in sensitivity of 0.76 (76\%) and specificity of 1 (100\%). The positive predictive value (PPV) was $1(100 \%)$, while the negative predictive value (NPV) was 0.676 (67.6\%). The accuracy of the parameter was $84 \%$ (Table 3 ).

\section{Discussion}

OSMF is of special concern not only due to the crippling condition that it produces in the patient but also because the entire mucosa acquires malignant potential. The prevalence of this condition is very high in the Indian population. In OSMF, once the fibrotic bands are palpable, the diagnosis become obvious on clinical examination, and the biopsy is done just for confirmation. However, for early 
diagnosis before blanching appears or the bands become properly palpable, the clinical examination may not be diagnostic because it is subjective in nature. Moreover, there are no known markers available for early diagnosis of OSMF. Also, the affected oral mucosa acquires potential to transform into malignancy, but because of reduced mouth opening a clinical examination as well as biopsy is difficult and early diagnosis of malignancy is not possible [10].

USG is based on the principle of an inverse piezoelectric effect [4]. USG is a non-invasive, real-time, nonionising, comfortable, less expensive technique that is suitable for visualisation of changes in the superficial structures like buccal and labial mucosa. It can examine a larger area in less time. The diagnostic potential of USG has been reported by Shetty et al. [11] and Sureshkannan et al. [12] in metastatic cervical lymph nodes, Tiwari et al. [13] in periapical pathologies, Sharma et al. [14] in facial space infection, Gandhi et al. [15] in OSCC, and Kundu et al. [16] in TMJ disorders. Despite these advantages of USG, very few studies have been conducted on USG in OSMF patients. Thus, the present study was conducted with the aim to evaluate the role of USG in OSMF.

In the present study, the mean submucosal thickness of right and left buccal mucosa and upper and lower labial mucosa increased significantly in Group II and in OSMF groups as compared to controls. The study results between control and OSMF groups are in accordance with Tiwari et al. [10], who reported increased submucosal thickness of right and left buccal mucosa and upper and lower labial mucosa in OSMF.

The present study partially favours Rangaiah et al. [5], who reported increased submucosal thickness at anterior and posterior buccal mucosa and upper and lower labial mucosa in OSMF cases as compared to controls but found no significant relationship between USG and clinical findings because the subjects attributed were greater in number in clinical stage III 10 (50\%) and IVa 4 (20\%). Also, Devathambi et al. [9] reported a significant increase in submucosal thickness with the advancement of stage of OSMF, but when the different stages were compared there was no statistical significant difference between stage II and III OSMF but significant correlation of stage II with stage IVa and stage III with stage IVa.

Similar study results were reported by Kumar et al. [17], who found a significant increase in submucosal thickness in OSMF cases compared to controls but failed to elucidate statistical significance across the stages and USG findings because there was heterogeneous distribution of the number of cases across the stages. In the present study there was significant correlation amongst OSMF stages and USG findings, which may be attributed to homogenous distribution of numbers in all groups of OSMF.

In the present study, the echogenicity pattern reported on USG in cases showed areas of irregular hyperechoic linear streaks due to increased fibrous deposits in the submucous layer, whereas the submucosa of the control group appeared hypoechoic band, which in is accordance with the study conducted by Krithika et al. [6] and Thapasum et al. [18], who demonstrated and delineated the junction between the hypoechoic submucosal bands and the muscle layer.

Vascularity plays a significant role in the treatment prognosis of OSMF. Poor vascularity in the affected region may respond poorly, and more than average vascularity may suggest malignant changes in the present study; peripheral arterial PSV in the submucosal band was assessed in colour Doppler spectral mode of buccal and labial mucosa. PSV was evaluated in the past in various inflammatory and neoplastic conditions. Li et al. [7], who performed USG in cases of scleroderma and reported decreased blood flow, increased echogenicity with increased fibrosis, and Gandhi et al. [15], who reported increased mean PSV in patients with malignancy compared to healthy subjects. The increased PSV in malignancy cases was because of the angiogenesis resulting in increased lesion vascularity. The mean PSV of right and left buccal mucosa and upper and lower labial was reduced significantly in Group II and OSMF groups as compared to Group I (Table 2). The comparison of mean arterial PSV across groups for both buccal and labial mucosa was highly significant except patients in OSMF stage III and IVa.

This reduced PSV from control to stage III OSMF showed clinical correlation with the degree of fibrosis and severity of condition. This is similar to the only study in the literature by Manjunath et al. [4], who performed intra-cavitary pre- and post-treatment ultrasonographic PSV evaluation in OSMF patients. They found that USG demonstrated fibrous bands and a pattern of overall vascularity in the affected area. Vascularity of mucosa overlying the band was found to be decreased with reduced flow velocity, while between the bands it was normal. They found that the USG peripheral venous PSV value was helpful in monitoring treatment prognosis and altering the treatment schedule if needed.

The sensitivity and specificity of USG in OSMF and control groups was not assessed in previous studies, but there is adequate literature available supporting the diagnostic accuracy of USG in maxillofacial pathologies like periapical lesions, space infections, temporomandibular joint (TMJ) disorders, lymph node metastasis, and oral malignancies $[8,13-16,19]$.

In the present study, the sensitivity and specificity of USG was evaluated in OSMF cases and controls, to assess its role in OSMF. The submucosal thickness of Group I and II was below the cut-off value, suggesting that although the thickness was significantly increased in Group II compared to controls, it cannot be considered to be of diagnostic importance. Submucosal thickness cut-off values of more than 1.37 for buccal mucosa and 1.47 for labial mucosa suggest that USG is an efficient tool in the diagnosis of OSMF according to present study. But out of 100 clinically diagnosed OSMF cases, only $80 \%$ showed buccal 
submucosal thickness more than the cut-off value (1.37), while $76 \%$ showed labial mucosa submucosal thickness more than the cut-off value (1.47). Thus, USG showed $87 \%$ and $84 \%$ accuracy in the diagnosis of OSMF using buccal and labial submucosal thickness findings, respectively (Table 3).

The PSV parameter was analysed by averaging the left and right buccal mucosa, as well as upper and lower labial mucosa. For either data set, the obtained AUC was 0.01, indicating that the PSV failed in classifying the cases from the controls, so this is not a reliable tool for diagnosis of OSMF. The diagnostic accuracy of USG was studied by Hayashi et al. [20], Tiwari et al. [13], Kundu et al. [16], Sharma et al. [14], Gandhi et al. [15], Shetty et al. [11], and Sureshkannan et al. [12], which involves hard and soft tissue lesions. Various authors, including Shetty et al. [11] and Sureshkannan et al. [12] performed the USG evaluation of cervical lymph nodes in head and neck cancer. The reported sensitivity, specificity, PPV, NPV, and accuracy by Shetty et al. [11] was $54.5 \%, 85.71 \%, 60 \%, 82.75 \%$, and $76.92 \%$, respectively, while by Sureshkannan et al. [12] it was $85.7 \%, 90 \%, 92.3 \%, 81.8 \%$, and $87.5 \%$, respectively. The present study results showed greater sensitivity as compared to specificity. The accuracy was also found to be higher as compared to the abovementioned studies. The sensitivity and accuracy of the present study results also support the study conducted by Gandhi et al. [15], who performed USG Doppler evaluation in OSCC cases and reported sensitivity $86.60 \%$ and accuracy $93.33 \%$.

The variations in sensitivity, specificity, PPV, NPV, and accuracy of diagnostic potential of USG may be due to variations in the sample size, different nature of orofacial pathologies, site of orofacial pathology (superficial or deep), as well as US operator variability. Kundu et al. [16] stated in their article that interpretation of USG images is highly dependent on the operator. Thus, well-trained and experienced operators are essential to get reliable results. USG can provide confirmatory evidence of fibrosis non-invasively. It takes less time to study larger area and shows differences between various soft tissues. USG can effectively differentiate normal from cystic, benign, and malignant lesions. It can show early changes of the condition in clinically healthy appearing mucosa in individuals with a habit. Fibrous bands can be studied in relation to vascularity and blood flow in regions in which it would not be possible in routine histopathological procedures. The treatment plan can be scheduled according to the vascularity of the region, and prognosis during treatment can be assessed.

\section{Conclusions}

In conclusion, it can be said that a disease such as OSMF, in which the severity varies site to site, requires a multimodal approach for effective assessment, management, and follow-up. In addition to the established methods of clinical and histopathological evaluation, USG provides a whole new third dimension by permitting tissue characterisation over the entire buccal and labial mucosa, indicating the depth of fibrosis, which is not possible by other methods. USG is a valuable, non-invasive, affordable, and radiation-free diagnostic tool that is easily accepted by patients, and it is less time consuming. We reported that as the severity of the disease advances, it showed a direct relation with submucosal thickness and an inverse relation with PSV. Also, habit-induced mucosal alteration in submucosal thickness can be seen on USG, which cannot be visualised on clinical examination. USG may be a better diagnostic tool in individuals with habits. Hence, USG can be a promising tool for diagnosis, for assessment of severity, and for evaluation of prognosis of OSMF.

\section{Acknowledgements}

The authors would like to thank Dr. Pawan Motghare, Dr. Anuraag Chaudhary, Dr. Tapasya Karemore, Dr. Ashita Kalaskar, Dr. Kajal Mitra, Dr. Sonam Heda, Dr. Rohit Biranjan, Dr. Sunil Baghel, Dr. Pallavi Assudani, Dr. Pritam Khorgade for their support and assistance.

\section{Conflict of interest}

The authors report no conflict of interest.

\section{References}

1. Murgod VV, Kale AD, Angadi PV, Hallikerimath S. Morphometric analysis of the mucosal vasculature in oral submucous fibrosis and its comparison with oral squamous cell carcinoma. J Oral Sci 2014; 56: $173-178$.

2. Rajendran R. Oral submucous fibrosis: etiology, pathogenesis, and future research. Bull World Health Organ 1994; 72: 985-996.

3. Kumar BS, Mahabob MN. Ultrasound in dentistry - a review. Journal of Indian Academy of Dental Specialist 2010; 1: 44-45.

4. Manjunath K, Rajaram PC, Saraswati TR, et al. Evaluation of oral submucous fibrosis using ultrasonographic technique: A new diagnostic tool. Indian J Dent Res 2011; 22: 530-536.

5. Rangaiah P, Annigeri R, Lingappa A. Transcutaneous ultrasonography assessment of oral submucous fibrosis: A preliminary Study. J Oral Med Sci 2010; 9: 137-147.

6. Karthika C, Ramanathan S, Koteeswaran D, et al. Ultrasonographic evaluation of oral submucous fibrosis in habitual areca nut chewers. Dentomaxillofac Radiol 2013; 42: 20120319.

7. Li SC, Liebling MS, Haines KA. Ultrasonography is a sensitive tool for monitoring localized scleroderma. Rheumatology 2007; 46: 1316-1319.

8. Sharma S, Rasila D, Singh M, Mohan M. Ultrasound as a diagnostic boon in dentistry - a review. Int J Sci Stud 2014; 2: 70-76. 
9. Devathambi JR, Aswath N. Ultrasonographic evaluation of oral submucous fibrosis and masseteric hyperthophy. J Clin Imaging Sci 2013; 3: 12 .

10. Tiwari M, Gupta M, Ghom S, Devi B. Ultrasonography evaluation of oralsubmucous fibrosis patient: A preliminary study. International Journal of Women Dentists 2014; 1: 1-4.

11. Shetty D, Jayade BV, Joshi SK, Gopalkrishan K. Accuracy of palpation, ultrasonography, and computed tomography in the evaluation of metastatic cervical lymph nodes in head and neck cancer. Indian J Dent 2015; 6: 121-124.

12. Sureshkannan P, Vijayprabhu, John R. Role of ultrasound in detection of metastatic neck nodes in patients with oral cancer. Indian J Dent Res 2011; 22: 419-423.

13. Tiwari B, Krishan M, Popli M, et al. Ultrasonography and Color Doppler in the Differential Diagnosis of Periapical Cyst and Granuloma. J Contemp Dent 2014; 4: 17-21.

14. Sharma M, Patil K, Guledgud MV. Ultrasonographic evaluation of facial space infections of odontogenic origin. J Oral Maxillofac Radiol $2014 ; 2: 8-14$
15. Gandhi R, Bhowate R, Nayyar AS, et al. Color Doppler ultrasonography in oral squamous cell carcinoma: Making ultrasonography more meaningful. Adv Biomed Res 2016; 5: 29.

16. Kundu H, Basavaraj P, Kote S, et al. Assessment of TMJ disorders using ultrasonography as a diagnostic tool: A review. J Clin Diagn Res 2013; 7: 3116-3120.

17. Kumar KS, Rastogi T, Kumar R, et al. Role of ultrasonography in oral submucous fibrosis. Bhavnagar University's Journal of Dentistry 2015; $1: 27-32$.

18. Thapasum, AF, Rangdhol, V, Mohammed F, et al. Gray-scale ultrasonographic imaging of the buccal mucosa in various stages of oral submucous fibrosis. Oral Radiol 2015; 31 : 143.

19. Pandya S, Chaudhary AK, Singh M, et al. Correlation of histopathological diagnosis with habits and clinical findings in oral submucous fibrosis. Head Neck Oncol 2009; 1: 10.

20. Hayashi T. Application of ultrasonography in dentistry. Japanese Dent Sci Rev 2012; 48: 5-13. 